\title{
Inferior Level of Maxillary Sinus and Cortical Bone Thickness at Maxillary Posterior Quadrant, in Three Different Growth Patterns: 3D-Computed Tomographic Study
}

\author{
Pavankumar Janardan Vibhute ${ }^{1,2}$ and Pushkar A. Patil ${ }^{3}$ \\ ${ }^{1}$ Department of Orthodontics, Yogita Dental College and Hospital, Khed, District Ratnagiri, Maharashtra 415709, India \\ 2"Braces \& Implant Clinic", 202-203, 4th Floor, Vishal Ventila, Near Noble Hospital, Hadapsar, Pune 411028, India \\ ${ }^{3}$ Department of Orthodontics, Sharad Pawar Dental College, Datta Meghe Institute of Medical Sciences, \\ Wardha, Maharashtra 442004, India
}

Correspondence should be addressed to Pavankumar Janardan Vibhute; drpavanvibhute@gmail.com

Received 5 September 2014; Accepted 20 November 2014; Published 15 December 2014

Academic Editor: Athanassios Kyrgidis

Copyright (C) 2014 P. J. Vibhute and P. A. Patil. This is an open access article distributed under the Creative Commons Attribution License, which permits unrestricted use, distribution, and reproduction in any medium, provided the original work is properly cited.

\begin{abstract}
Objective of this retrospective study was to measure cortical bone thickness and lowest level of maxillary sinus at maxillary posterior quadrant in different growth pattern (hypodivergent, average, and hyperdivergent) at prospective microimplant placement sites in order to understand both safety and stability aspects of microimplant placement by using cone-beam 3-dimensional computed tomographic images. In posterior quadrant, vertical distance from cementoenamel junction to lowest level of maxillary sinus at interradicular region was measured. Buccal cortical bone thickness was measured at 3 different vertical levels at interradicular space. The cortical bone thickness was found more at sinus floor level and above it than below the sinus floor. In perspective of miniscrew placement, study shows that maxillary sinus floor is safer with average and hyperdivergent growth pattern than hypodivergent growth pattern. In the proximity of maxillary sinus floor, $1 \mathrm{~mm}$ or more cortical bone can be expected in maxillary posterior region in average and hyperdivergent growth patterns. However, it was thicker in hypodivergent than hyperdivergent growth patterns. Hypodivergent patients require either more horizontal insertion (more parallel to occlusal plane) or shorter length of miniscrew to avoid damage to maxillary sinus.
\end{abstract}

\section{Introduction}

Mini-implants have become the popular source of orthodontic anchorage, because they can be placed in various locations in the alveolar bone [1-4]. Several investigators have recommended, in posterior maxilla, the zygomatic crest specifically, the buccal surface of the malar process for skeletal anchorage during retraction of maxillary anterior teeth, intrusion of posterior teeth, and other orthodontic movements. This site is preferable to miniscrew insertion because of its two cortical layers (buccal and sinus floor) which ensure primary stability if a screw of appropriate length is fixed bicortically $[5,6]$. The primary challenge to safe insertion of miniscrews in the zygomatic crest is the possibility of perforating the
Schneiderian membrane and penetrating the antrum of the maxillary sinus [7]. There is also no consensus on use of sinus wall cortex for miniscrew stability.

It is known that the quantity (bone volume) and quality (bone density) of alveolar bone are important factors for the stability of miniscrew [8]. Structurally, the maxilla has relatively thin cortices that are interconnected by a network of trabeculae [9]. Cortical bone morphology is influenced by force application and also influenced by the stresses and strain produced by functional loads of associated muscle of mastication. Thus, anatomical characteristics such as the thickness of cortical bone might differ between the different skeletal patterns. By angulating the miniscrew, the thickness of cortical bone contact with the miniscrew might increase 
but might result in maxillary sinus damage $[8,10]$. In addition, we previously suggested that cortical bone thickness might have some effect on implant success rate.

Few studies have evaluated the quantity of cortical bone in maxilla and mandible for implant placement for orthodontic anchorage [11-13], but none of these studies shows the cortical bone thickness in the proximity of maxillary sinus. It is also important to determine the lower level of maxillary sinus in different growth pattern, to avoid complication of sinus damage during miniscrew placement. Studies have warned of possible presence of the sinus in these areas often selected for screw placement in relation to tooth roots $[10,14]$. Poggio et al. [10] reported that the insertion of miniscrews in the maxillary molar region above $8 \mathrm{~mm}$ from the alveolar crest must be avoided because of the presence of the sinus but also these studies lack the difference in the level of maxillary sinus in different skeletal growth pattern individuals.

Thus, the purpose of this computed tomographic (CT) image study was to determine the lower level of maxillary sinus and buccal cortical bone thickness in maxillary posterior quadrant in different growth pattern individual, from perspective of safe placement and increased primary stability.

\section{Materials and Methods}

30 untreated adult patients with bimaxillary protrusion and fairly aligned arches of age ranges between 18 and 25 years (mean age 22.4 years) with no craniofacial anomalies or systemic disease were selected. Patients with severe crowding, missing teeth, or radiographic signs of periodontal disease were excluded. They were divided into three groups based on Frankfurt mandibular plane angle (FMPA) [15], 10 hypodivergent (FMPA less than $16^{\circ}$ ), 10 hyperdivergent (FMPA above $34^{\circ}$ ), and 10 average growth pattern (FMPA between $22^{\circ}$ and $27^{\circ}$ ) cases. Each patient was scanned on KODAK 9000C 3D machine (Trophy, France) which is in compliance with the requirements of the EEC (European Economic Community) and International Medical standards at $70 \mathrm{Kv}$ and $10 \mathrm{~mA}$ for 10.8 seconds for each quadrant of the jaw. The added power of a $50 \mathrm{~mm} \times 38 \mathrm{~mm}$ focused field of view helps the operator to visualize specific regions of interest with true anatomic accuracy.

The CT images were formatted into standard DICOM and reconstructed into continuous slices at $0.76 \mu \mathrm{m}$ thickness each. (Figures 1(a) and 1(b)) The CT image analysis for each image was conducted by Kodak 3D viewer, 2.2 version software, and curved slicing images with sections of $76 \mu \mathrm{m}$ thickness were chosen for measurements (Figure 2). Same person to minimize human error obtained all measurements.

Procedure to measure LLMS is as follows. Line was drawn at superior border of the most inferior point on the floor of the maxillary sinus. Second line was drawn from the mesial CEJ of one tooth to the distal CEJ of adjacent tooth and perpendicular distance between these two lines was taken as the lower level of maxillary sinus from CEJ. First LLMS measurement was done on buccal side between the 1st premolar and 2nd molar region in the interradicular space, from CEJ on both sides of the maxilla (Figures 3(a) and 3(b)).
Procedure to measure CBT is as follows. Buccal CBT measurement was done at the interradicular space between 2nd premolar-1st molar and 1st molar-2nd molar at 3 different levels, that is, at LLMS, $2 \mathrm{~mm}$ above LLMS, and $2 \mathrm{~mm}$ below LLMS. Each measurement was taken from the buccal alveolar plate. For this measurement, a reference horizontal line was drawn at the lowest level of sinus floor parallel to CEJ and three horizontal measurements were taken parallel to this line at 3 different vertical levels (Figures 4(a) and 4(b)).

\section{Results}

In hyperdivergent and average growth pattern LLMS ranged from 11.11 to $20.86 \mathrm{~mm}$ (SD, 3.02-5.06) and 10.04 to $16.21 \mathrm{~mm}$ (SD, 1.98-2.49), respectively; in both groups the LLMS tend to get decreased from premolar segment to molar segment and LLMS was found least between 1st and 2nd molar and greatest between 1st and 2nd premolar. In hypodivergent growth pattern, average LLMS ranged from 6.3 to $11.22 \mathrm{~mm}$ (SD, 0.99-2.05). LLMS was least between 2nd premolar and 1st molar, whereas between 1st and 2nd premolar it was greatest. In general, LLMS was least in hypodivergent compared to hyperdivergent and average growth pattern (Figure 5, Table 1).

Mean CBT in hypodivergent growth pattern ranged from 1.51 to $2.97 \mathrm{~mm}$ (SD 0.18-0.74). The CBT was found more between 2 nd premolar and 1st molar at the LLMS and least between 2nd premolar and 1st molar below LLMS (Table 2). In average growth pattern CBT ranged from 1.03 to $1.57 \mathrm{~mm}$ (SD 0.11-0.43); CBT was found more between 1st and 2nd molar above LLMS and least between 2nd premolar and 1st molar below LLMS (Table 3). In hyperdivergent growth pattern, average CBT was ranged from 0.9 to $2 \mathrm{~mm}$ (SD 0.12-0.36). More CBT was found between 1st and 2nd molar at LLMS and least between 2nd premolar and 1st molar below LLMS (Table 4). In general, the CBT was more in hypodivergent growth pattern and least in hyperdivergent growth pattern and it was more at and above the LLMS than below LLMS in all 3 groups.

\section{Discussion}

Miniscrew placement technique is an artistic performance aided at most by conventional 2-dimensional X-ray images. Most miniscrews are currently placed high in the interradicular spaces between 2nd premolar and 1st permanent molar or between 1st and 2nd permanent molar in order to avoid root damage without 3-dimensional information of bone thickness and maxillary sinus floor level. This placement protocol offers little protection against the 2 major problems of miniscrew placement, that is, the possibility of maxillary sinus damage and the danger of premature loosening of the implant due to inadequate primary stability [16]. Therefore, the aim of this study was to evaluate the variation in cortical bone thickness and level of maxillary sinus and their correlation with different growth patterns in the maxillary posterior regions by using $3 \mathrm{D} \mathrm{CT}$.

Safe placement zone and initial stability are the keys to the overall success of miniscrew anchorage. Without initial 


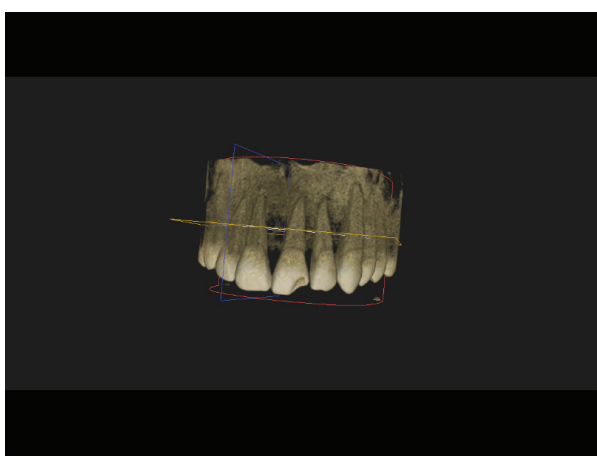

(a)

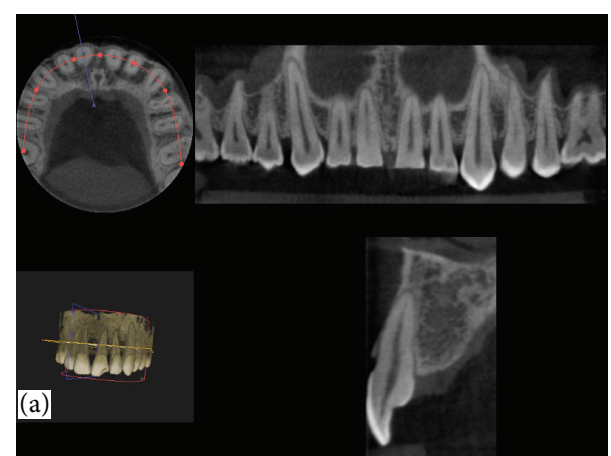

(b)

FIGURE 1: (a) 3D reconstruction of maxilla. (b) Orientation of maxilla in 3 planes.

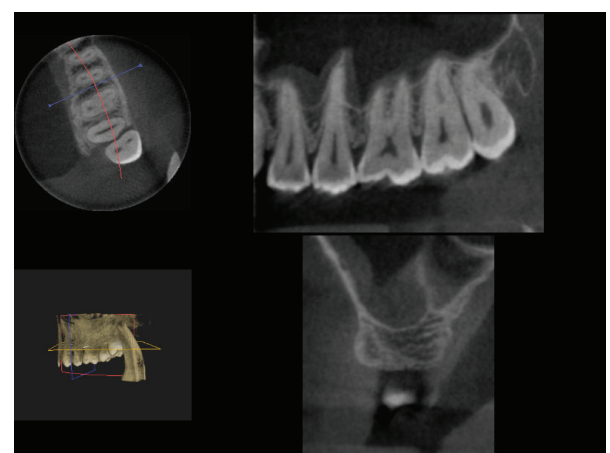

Figure 2: One quadrant oriented to measure LLMS from CEJ and CBT between 1st and 2nd molar interdentally.

stability, there will not be a late stability. Initial stability is derived from good mechanical interlocking between the implant and the bone.

Initial stability is influenced by 3 factors, bone quality (host factor), implant design (material factor), and placement technique (operator factor). For bone quality, cortical bone thickness is the most important determinant of initial stability [16].

As demonstrated by numerous studies, cortical bone thickness of the maxilla and mandible varies. The pattern of cortical bone thickness appears to be somewhat consistent from one individual to another. In general, the cortex increases in thickness from molar segment to premolar segment and is thicker on the palatal side of the maxilla than the buccal side, and in the mandible, it is thicker on the buccal aspect as compared to the lingual aspect. If the patterns are generally consistent, then what is the cause for this variation?

To understand these patterns, a discussion of the function of bone is necessary. One of the functions of bone is to support the structures around it. Bone must be stiff and strong enough to resist the stress and strain developed. Additionally, it must be able to adapt to the changes due to growth and the environment. The stress and strain experienced by bone include axial compression, bending, twisting, and shearing. Bone is able to withstand the stress and strain up to a point.
The point at which permanent deformation occurs on a stress-strain curve is known as the yield point. The fracture point is the point beyond the yield point at which the bone breaks. The region of the stress-strain curve that pertains to the present project is between the yield point and the ultimate tensile strength point. Bone modeling and remodeling occurs based on the applied stresses and strains.

With respect to the muscles of mastication, the weaker the musculature is, the weaker the bite forces are. Weak bite forces lead to smaller functional effects on the maxilla and mandible.

Miyajima et al. [17] and Dalstra et al. [18] showed maximum stress at the cortical bone level when an implant is loaded. In a finite element study, Dalstra et al. [18] demonstrated that increasing cortical bone thickness drastically reduced the peak strain development in the peri-implant bone tissue. In particular with $1 \mathrm{~mm}$ or more of cortical thickness, the peak strain in the bone was confined to the adapted window of Frost's Mechanostat theory [19-22]. This inverse relationship between cortical bone thickness and peak strain development suggests that cortical bone thickness is the key determinant of initial stability.

Miyamoto et al. [23] used resonance frequency analysis to measure the initial placement torque of restorative implants. They quantified initial stability by units of implant stability quotient (ISQ) derived from resonance frequency analysis value. Using linear regression analysis, they also showed a high correlation between cortical bone thickness and the ISQ value (r 5 0.84). This close relationship between initial stability and cortical bone thickness was similar to the findings of Wilmes et al. [24]. Miyamoto et al. concluded that implant stability at placement largely depends on local bone conditions [23].

The importance of cortical bone thickness was again demonstrated by a study of Wilmes et al. [25]. They found placing microimplants at an angle between $60^{\circ}$ and $70^{\circ}$ resulted in the highest placement torque values. At an oblique angle, the microimplant can obtain a longer distance through cortical bone and achieve higher initial stability.

Results of our study showed significant differences in cortical bone thickness in the proximity of maxillary sinus of the hypodivergent individuals, when compared to normal 


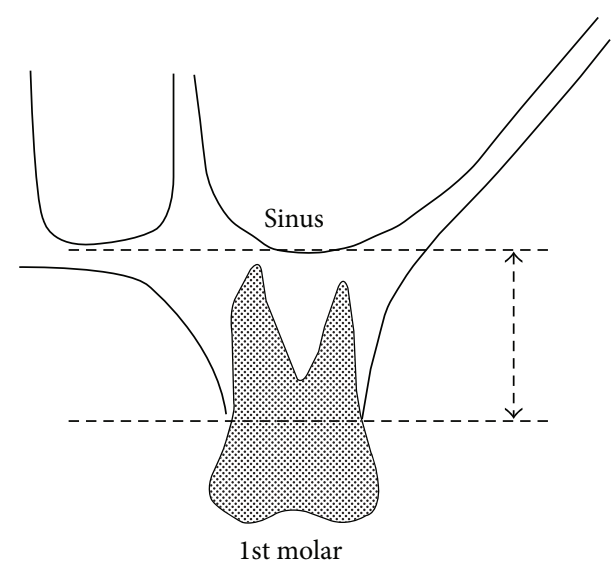

(a)

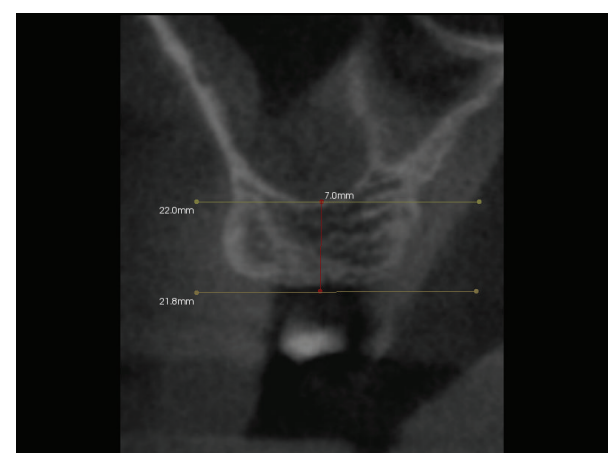

(b)

FIgURE 3: (a) Schematic diagram showing LLMS measurement. (b) Actual measurement of LLMS.

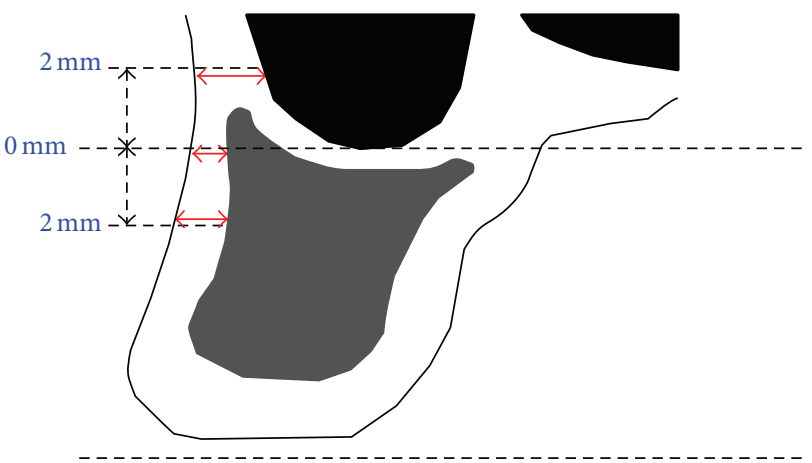

(a)

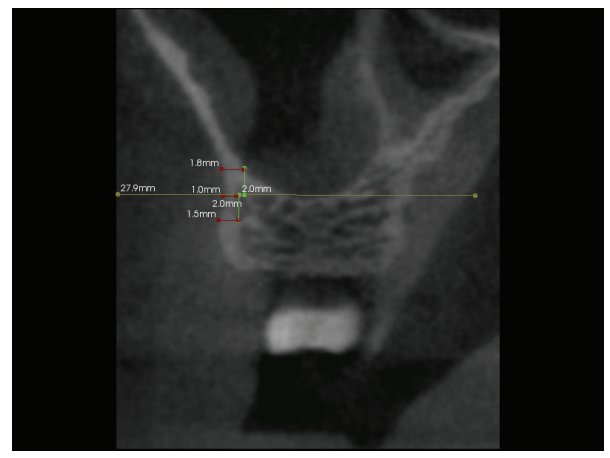

(b)

Figure 4: (a) Schematic CBT measurement. (b) Actual measurement of CBT.

and hyperdivergent growth pattern individuals. Present study shows, cortical bone thickness in hypodivergent growth pattern individuals is thicker than that of hyperdivergent and normal growth pattern individuals. Similar results were found in 1998 by Tsunori et al. [26] and in 2001 by Masumoto et al. [27]. They found that the cortical bone thickness of the first and second molar section was thicker in short face subjects by $0.1-2.5 \mathrm{~mm}$ than the normal and long face subjects. From the present study, similar finding was obtained for the cortical bone thickness at 2 nd premolar and 1st permanent molar section was thicker in hypodivergent subject by 0.1 to $2 \mathrm{~mm}$ and at 1 st and 2nd permanent molar section by 0.4 to $1.7 \mathrm{~mm}$ than hyperdivergent and normal growth pattern individuals. The differences of cortical bone thickness may be explained by masticatory function and its corelation with the facial type.

In the present study it was found that the cortical bone thickness in the proximity to maxillary sinus in hypodivergent individuals was more than $1.5 \mathrm{~mm}$ irrespective of interradicular site while the cortical bone thickness at some sites of hyperdivergent subjects was less than $0.8-0.9 \mathrm{~mm}$. It has been suggested that miniscrew success depends on placing it in at least $1 \mathrm{~mm}$ thickness of cortical bone [28]. This raises question about miniscrew implant placement in individuals with hyperdivergent growth pattern.

Park and Cho [16] state that cortical bone thickness was site dependent and it increased as the distance from the alveolar ridge increased. In the present study, similar findings were found. The thickness of buccal cortical bone was more at the level of and $2 \mathrm{~mm}$ above the LLMS (i.e., coronal to sinus), as compared to $2 \mathrm{~mm}$ below the LLMS (i.e., apical to sinus). This difference may be because of presence of two cortical layers (buccal and sinus floor) at and coronal to LLMS.

In the present study, sex differences were not studied since prior studies have shown no sex differences in cortical bone thickness of either the maxilla or mandible [8]. Since males and females eat essentially the same types of food, the strain produced during mastication might be expected to be similar, as would cortical bone thickness.

While it is generally accepted that some degree of anatomic asymmetry is widely displayed in the normal human craniofacial complex, this study failed to find a generalized significant difference $(P>0.05)$ between the right and left sites measured. 


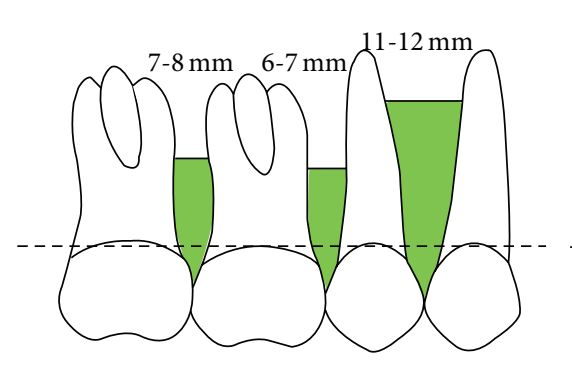

Hypodivergent growth pattern

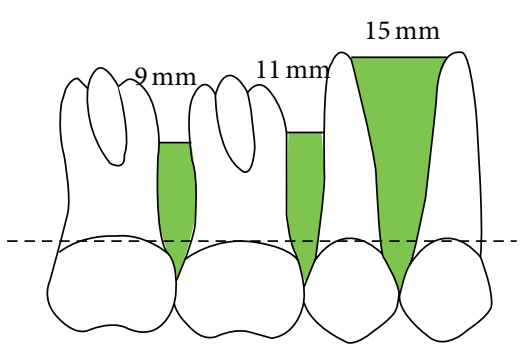

Average growth pattern

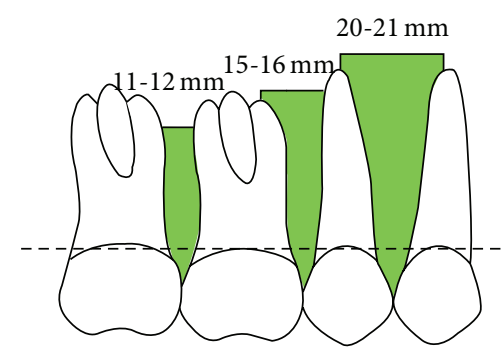

Hyperdivergent growth pattern

FIGURE 5: LLMS comparison between hypodivergent growth pattern, average growth pattern, and hyperdivergent growth pattern.

4.1. Safe Zone for Miniscrew Placement in Posterior Maxilla with respect to Maxillary Sinus. Previously, interradicular sites were analyzed for the safe placement of miniscrew in posterior maxilla to avoid root damage $[10,12,16]$. The general recommendation is to place miniscrews in attached gingiva $[12,29,30]$, but apical placement is favored since the interradicular distance increases in the apical direction which reduces the risk of root damage $[12,16]$. Inserting miniscrew at an angle directed apically will lead to an increased primary bone-to-implant contact $[8,10]$. On the other hand, this might increase the risk of sinus perforation. In particular, the risk of sinus perforation with interradicular miniscrews placed from the buccal aspects with an insertion directed apically has not been addressed systematically, so far.

The risk of perforation of the maxillary sinus during miniscrew insertion has merely been reported in relation to insertion at the apical level of the molars, including the infrazygomatic crest, and distally to the second molar [10, 3133].

Long miniscrews of 8 to $10 \mathrm{~mm}$ have been reported to be associated with a higher risk of sinus membrane perforation than the shorter miniscrews of $6 \mathrm{~mm}$ [34].

Thus, from the previous studies it has been concluded that the length of miniscrew as well as more apical or angulated placement of miniscrews results in perforations of maxillary sinus.

Previous studies showed the differences in the level of maxillary sinus and gave different limit for placement of miniscrews in the individuals $[10,14]$ but failed to specify the reason for this variability.

As the musculature influences the anatomical development of face, maxilla, and mandible, there might be an anatomical variation associated with development of paranasal sinuses as muscle function varies. To see this anatomical variation, the lower level of maxillary sinus was analyzed in different growth pattern individuals for safe placement of miniscrew.

Thus, in order to characterize the relationship of the maxillary sinus and the CEJ of teeth in posterior maxilla as well as clarifying any morphological differences between various interradicular sites and different growth patterns individuals, analysis was carried out with 3D CT of posterior maxilla in hypodivergent, hyperdivergent, and normal growth pattern.
In the present study it was found that in hyperdivergent and normal growth pattern individuals the maxillary sinus level drops gradually from 1st premolar to 2 nd permanent molar and most inferior level was found between 1st and 2nd permanent molar. Similar findings were observed by Kim et al. [35], who studied the maxillary sinus thickness with the help of CT scan. They concluded that the maxillary sinus floor was located most inferiorly between the 1st molar and 2nd molar and most superiorly between the 1st premolar and 2nd premolar. But the finding for hypodivergent individuals is contradictory to their finding. In this group the maxillary floor was located more inferiorly between 2 nd premolar and 1st permanent molar region.

Results of this study showed significant differences in the level of maxillary sinus in hypodivergent individuals, when compared to normal and hyperdivergent individuals. These differences in the level might be because of the difference in muscular pattern, which has an influence on anatomical development of face.

Previous studies recommended that the interradicular sites mesial to maxillary 1st molar slight apical to the midroot level are safe sites for insertion of miniscrews $[8,10,16]$, and sinus perforations are expected to be a relatively common side effect. Nevertheless, not much attention has been given to this side effect, presumably because a small perforation rarely creates complications and heals without intervention [36-38].

In the present study, relative short distance of maxillary sinus from CEJ in hypodivergent groups implies a risk of perforation of the maxillary sinus if miniscrews are planned for insertion with an apical inclination. The normal distance of maxillary sinus floor at interradicular site between 2nd premolar and 1st permanent molar was $6.30 \mathrm{~mm}$ and $6.90 \mathrm{~mm}$ on right and left side, respectively, suggesting that maxillary floor extended up to midroot level in this group, so miniscrew above this level should be inserted cautiously. Similar limit was suggested by Ishii et al. [14] in their study. They recommended miniscrew placement is not higher than $6-8 \mathrm{~mm}$ from the alveolar crest due to presence of maxillary sinus.

Poggio et al. [10] provide an anatomical map to assist the clinician in miniscrew placement in a safe location between dental roots. They advocate insertion of the miniscrews in the 
TABLE 1: Distance of LLMS from CEJ (mean value, in millimeters).

\begin{tabular}{lccccccc}
\hline & & R 4-5 & R 5-6 & R 6-7 & L 4-5 & L 5-6 & L 6-7 \\
\hline \multirow{2}{*}{ Hypodivergent growth pattern } & Mean & 11.03 & 6.3 & 7.77 & 11.22 & 6.9 & 7.45 \\
& SD & 1.46 & 1.48 & 1.66 & 2.05 & 1.75 & 0.99 \\
\hline \multirow{2}{*}{ Average growth pattern } & Mean & 16.21 & 12.35 & 10.04 & 15.47 & 11.94 & 10.82 \\
& SD & 1.98 & 2.36 & 2.34 & 2.49 & 2.37 & 2.48 \\
\hline \multirow{2}{*}{ Hyperdivergent growth pattern } & Mean & 20.0 & 14.92 & 11.11 & 20.86 & 15.11 & 11.34 \\
& SD & 3.68 & 3.17 & 3.29 & 5.06 & 3.26 & 3.02 \\
\hline
\end{tabular}

TABLE 2: Buccal CBT in hypodivergent (mean value, in millimeters).

\begin{tabular}{lcccrr}
\hline & & R 5-6 & R 6-7 & L 5-6 & L 6-7 \\
\hline \multirow{2}{*}{ CBT 2 mm above LLMS } & Mean & 2.19 & 2.53 & 2.36 & 2.61 \\
& SD & 0.53 & 0.70 & 0.29 & 2.57 \\
\hline \multirow{2}{*}{ CBT at LLMS } & Mean & 2.46 & 2.11 & 0.53 & 0.74 \\
\hline \multirow{2}{*}{ CBT 2 mm below LLMS } & SD & 0.22 & 1.68 & 1.66 & 0.36 \\
& Mean & 1.51 & 0.18 & 0.30 & 1.61 \\
\hline
\end{tabular}

TABLE 3: Buccal CBT average growth pattern (mean value, in millimeters).

\begin{tabular}{lcccrr}
\hline & & R 5-6 & R 6-7 & L 5-6 & L 6-7 \\
\hline \multirow{2}{*}{ CBT 2 mm above LLMS } & Mean & 1.36 & 1.57 & 1.46 & 0.43 \\
\hline \multirow{2}{*}{ CBT at LLMS } & SD & 0.22 & 1.50 \\
& Mean & 1.21 & 0.15 & 1.28 & 0.22 \\
\hline \multirow{2}{*}{ CBT 2 mm below LLMS } & SD & 0.23 & 1.14 & 0.17 & 1.34 \\
& Mean & 1.03 & 0.15 & 0.22 \\
\hline
\end{tabular}

maxillary molar region above $8-11 \mathrm{~mm}$ from the bone crest to be avoided because of the presence of the sinus. In present study similar limit was observed for individuals with normal growth pattern but this limit contradicts that observed for hypodivergent individuals.

In hyperdivergent individuals, high placement of miniscrew is required for vertical control and for intrusion of posteriors. Lower level of maxillary sinus was found farthest in this group at each interradicular space, compared to the rest of 2 groups of this study. Between 2 nd premolar and 1st permanent molar the maxillary sinus was at $15.5 \mathrm{~mm}$ and $15.11 \mathrm{~mm}$ on right and left side, respectively, and between 1st and 2nd permanent molar it was at $11.11 \mathrm{~mm}$ and $11.34 \mathrm{~mm}$ on right and left side, respectively.

From these findings upper limit for placement of miniscrew in hyperdivergent individuals was found to be 14$15 \mathrm{~mm}$ and $10-11 \mathrm{~mm}$, at interradicular space between 2 nd premolar-1st permanent molar and 1st-2nd permanent molar, respectively.

Similarly, in normal growth pattern individuals the upper limit for miniscrew insertion was found to be $11-12 \mathrm{~mm}$ and $9-10 \mathrm{~mm}$ at interradicular space between 2nd premolar-1st permanent molar and 1st-2nd permanent molar, respectively, which is similar to the limit given by Poggio et al. [10].
The use of long miniscrews $(8 \mathrm{~mm})$ is recommended in order to increase primary stability when thin cortical bone (less than $1 \mathrm{~mm}$ ) is expected $[18,28]$. In the maxillary molar region, insertion of $8 \mathrm{~mm}$ long miniscrew at $45^{\circ}$ to the long axis of the teeth will increase the cortical boneto-implant contact (compared to perpendicular insertion) and the trabecular bone-to-implant contact in cases with a sufficient distance from the sinus. Furthermore, the use of an $8 \mathrm{~mm}$ miniscrew will increase the chance of obtaining bicortical (buccal and sinus floor) anchorage, shown by Brettin et al. [39], which is superior to unicortical anchorage. However, apically angulated insertion in the maxillary molar region will lead to a higher risk of sinus perforation.

The present study indicates that the risk of sinus perforation can be reduced without compromising the primary stability in hypodivergent individuals, if the $8 \mathrm{~mm}$ miniscrew is inserted $6-7 \mathrm{~mm}$ from CEJ, where sufficient cortical bone was observed, similarly in hyperdivergent individuals when high placement is required apically and angulated miniscrew insertion (for attaining more contact of cortical bone) is found safe at $10-12 \mathrm{~mm}$ from CEJ.

This study failed to find a generalized significant difference $(P=0.05)$ between the left and right side. This would support the findings of Fernandes [40] who found no 
TABLE 4: Buccal CBT in hyperdivergent (mean value, in millimeters).

\begin{tabular}{lcccrr}
\hline & & R 5-6 & R 6-7 & L 5-6 & L 6-7 \\
\hline \multirow{2}{*}{ CBT 2 mm above LLMS } & Mean & 0.87 & 0.98 & 0.91 & 0.95 \\
& SD & 0.18 & 0.27 & 0.15 & 0.36 \\
\hline \multirow{2}{*}{ CBT at LLMS } & Mean & 0.87 & 1.20 & 1.02 & 0.15 \\
\hline \multirow{2}{*}{ CBT 2 mm below LLMS } & SD & 0.18 & 0.27 & 0.36 \\
& Mean & 0.84 & 0.95 & 0.96 & 0.21 \\
\hline
\end{tabular}

significant difference between left and right maxillary sinus volume and linear measurements in his sample of 53 dried skulls. In light of this finding, left and right measurements were combined in an effort to bolster the sample size for all further analyses.

Previous studies suggest that the palatal site provide more clearance from possible interference with maxillary sinus, and Ishii et al. [14] and Poggio et al. [10] also specifically recommend this site as the safest in the posterior maxillary alveolus. Therefore, in our study consideration was given only to the buccal placement site and relation of maxillary sinus was measured with CEJ at buccal side of posterior maxilla.

4.1.1. Clinical Implications. This study provides valuable information to the clinician with regard to preferable sites for miniscrew placement in order to avoid maxillary sinus damage and for primary stability amongst different growing pattern individuals by utilizing high resolution CT scanning.

In hypodivergent growth pattern, miniscrew insertion 6-7 $\mathrm{mm}$ above the CEJ should be avoided and when high placement is required, the miniscrew should be placed less angulated and more parallel to occlusal plane.

For vertical control and for intrusion of posterior teeth, high placement at $10-12 \mathrm{~mm}$ from CEJ is found to be safe in hyperdivergent individuals, but soft tissue and cortical bone thickness should be considered. In this group angulated insertion of miniscrew in order to achieve more cortical bone in this group might be helpful.

4.1.2. Limitations of Study. There were potential limitations to this study. Limited sample size of the present study was a limitation. Measurements were subjected to human error, and the direction of measurements has an influence on the measurement of the cortical bone thickness, as the surface is generally slightly angulated in relation to the long axis of the teeth. Cortical borders are often indistinct and require a certain amount of extrapolation by the measurer. This may artificially increase or decrease the cortical bone thickness. Bone mineral density was not evaluated in this study, which has an effect on primary stability of the implant. The study was performed only in age range of 18-26 years without missing teeth; however, it is also important to see the variations in maxillary sinus anatomy in the older individuals and in individuals with missing teeth.
4.1.3. Scope for Future Studies. In future research, it is recommended to evaluate bone mineral density of cortical bone although 2 cortical bones can have the same thickness, and they might have completely different bone mineral densities and hence different initial stability values. In the present method of evaluation, bone mineral density was not considered because it was not possible to calibrate the mineral density with the same software. In addition, more studies are needed to evaluate the anatomy of maxillary sinus among different ethnic groups, different dentoskeletal patterns, and dentofacial anomalies which is poorly documented in the literature.

\section{Conclusion}

From perspective of miniscrew placement, maxillary sinus floor is safer with average and hyperdivergent growth pattern than hypodivergent growth pattern. In the proximity of maxillary sinus floor, $1 \mathrm{~mm}$ or more cortical bone can be expected in maxillary posterior region; however, it is thicker in hypodivergent than hyperdivergent growth patterns. Hypodivergent patients require more horizontal insertion or length of miniscrew should be reduced.

\section{Conflict of Interests}

The authors declare that there is no conflict of interests regarding the publication of this paper.

\section{References}

[1] W. E. Roberts, C. L. Nelson, and C. J. Goodacre, "Rigid implant anchorage to close a mandibular first molar extraction site," Journal of Clinical Orthodontics, vol. 28, no. 12, pp. 693-704, 1994.

[2] M. Umemori, J. Sugawara, H. Mitani, H. Nagasaka, and H. Kawamura, "Skeletal anchorage system for open-bite correction," American Journal of Orthodontics and Dentofacial Orthopedics, vol. 115, no. 2, pp. 166-174, 1999.

[3] S. Kuroda, A. Katayama, and T. Takano-Yamamoto, "Severe anterior open-bite case treated using titanium screw anchorage," Angle Orthodontist, vol. 74, no. 4, pp. 558-567, 2004.

[4] A. Costa, M. Raffainl, and B. Melsen, "Miniscrews as orthodontic anchorage: a preliminary report," The International Journal of Adult Orthodontics and Orthognathic Surgery, vol. 13, no. 3, pp. 201-209, 1998. 
[5] E. J. W. Liou, P. H. Chen, Y. C. Wang, and J. C. Y. Lin, "A computed tomographic image study on the thickness of the infrazygomatic crest of the maxilla and its clinical implications for miniscrew insertion," The American Journal of Orthodontics and Dentofacial Orthopedics, vol. 131, no. 3, pp. 352-356, 2007.

[6] F. Khoury, "Augmentation of the sinus floor with mandibular bone block and simultaneous implantation: a 6-year clinical investigation," The International Journal of Oral \& Maxillofacial Implants, vol. 14, no. 4, pp. 557-564, 1999.

[7] A. Gracco, S. Tracey, and U. Baciliero, "Miniscrew insertion and the maxillary sinus: an endoscopic evaluation," Journal of Clinical Orthodontics, vol. 44, no. 7, pp. 439-443, 2010.

[8] T. Deguchi, M. Nasu, K. Murakami, T. Yabuuchi, H. Kamioka, and T. Takano-Yamamoto, "Quantitative evaluation of cortical bone thickness with computed tomographic scanning for orthodontic implants," The American Journal of Orthodontics and Dentofacial Orthopedics, vol. 129, no. 6, pp. 721.e7-721.e12, 2006.

[9] R. Adell, U. Lekholm, B. Rockler, and P. I. Branemark, "A 15year study of osseointegrated implants in the treatment of the edentulous jaw," International Journal of Oral Surgery, vol. 10, no. 6, pp. 387-416, 1981.

[10] P. M. Poggio, C. Incorvati, S. Velo, and A. Carano, "Safe zones': a guide for miniscrew positioning in the maxillary and mandibular arch," Angle Orthodontist, vol. 76, no. 2, pp. 191-197, 2006.

[11] S. Miyawaki, I. Koyama, M. Inoue, K. Mishima, T. Sugahara, and T. Takano-Yamamoto, "Factors associated with the stability of titanium screws placed in the posterior region for orthodontic anchorage," American Journal of Orthodontics and Dentofacial Orthopedics, vol. 124, no. 4, pp. 373-378, 2003.

[12] M. A. Schnelle, F. M. Beck, R. M. Jaynes, and S. S. Huja, "A radiographic evaluation of the availability of bone for placement of miniscrews," Angle Orthodontist, vol. 74, no. 6, pp. 832-837, 2004.

[13] A. Gahleitner, B. Podesser, S. Schick, G. Watzek, and H. Imhof, "Dental CT and orthodontic implants: imaging technique and assessment of available bone volume in the hard palate," European Journal of Radiology, vol. 51, no. 3, pp. 257-262, 2004.

[14] T. Ishii, K. Nojima, Y. Nishii, T. Takaki, and H. Yamaguchi, "Evaluation of the implantation position of mini-screws for orthodontic treatment in the maxillary molar area by a micro CT,' The Bulletin of Tokyo Dental College, vol. 45, no. 3, pp. 165$172,2004$.

[15] C. H. Tweed, "The frankfort-mandibular plane angle in orthodontic diagnosis, classification, treatment planning, and prognosis," American Journal of Orthodontics and Oral Surgery, vol. 32, no. 4, pp. 175-230, 1946.

[16] J. Park and H. J. Cho, "Three-dimensional evaluation of interradicular spaces and cortical bone thickness for the placement and initial stability of microimplants in adults," The American Journal of Orthodontics and Dentofacial Orthopedics, vol. 136, no. 3, pp. 314.e1-314.e12, 2009.

[17] K. Miyajima, S. Saito, M. Sana, and M. Sakai, "Threedimensional finite element models and animal studies of the use of mini-screws for orthodontic anchorage," The Angle Orthodontist, vol. 27, no. 2, pp. 111-115, 2005.

[18] M. Dalstra, P. M. Cattaneo, and B. Melsen, "Load transfer of miniscrews for orthodontic anchorage," Orthodontics, vol. 1, pp. 53-62, 2004.

[19] H. M. Frost, "Bone "mass" and the "mechanostat": a proposal," Anatomical Record, vol. 219, no. 1, pp. 1-9, 1987.
[20] H. M. Frost, "Skeletal structural adaptations to mechanical usage (SATMU): 1. Redefining Wolff's law: the bone modeling problem," Anatomical Record, vol. 226, no. 4, pp. 403-413, 1990.

[21] H. M. Frost, "Skeletal structural adaptions to mechanical usage (SATMU): 2. Redefining Wolff's law: the remodeling problem," Anatomical Record, vol. 226, no. 4, pp. 414-422, 1990.

[22] H. M. Frost, "Wolff's Law and bone's structural adaptations to mechanical usage: an overview for clinicians," Angle Orthodontist, vol. 64, no. 3, pp. 175-188, 1994.

[23] I. Miyamoto, Y. Tsuboi, E. Wada, H. Suwa, and T. Iizuka, "Influence of cortical bone thickness and implant length on implant stability at the time of surgery-Clinical, prospective, biomechanical, and imaging study," Bone, vol. 37, no. 6, pp. 776780, 2005.

[24] B. Wilmes, C. Rademacher, G. Olthoff, and D. Drescher, "Parameters affecting primary stability of orthodontic miniimplants," Journal of Orofacial Orthopedics, vol. 67, no. 3, pp. 162-174, 2006.

[25] B. Wilmes, Y.-Y. Su, and D. Drescher, "Insertion angle impact on primary stability of orthodontic mini-implants," The Angle Orthodontist, vol. 78, no. 6, pp. 1065-1070, 2008.

[26] M. Tsunori, M. Mashita, and K. Kasai, "Relationship between facial types and tooth and bone characteristics of the mandible obtained by CT scanning," Angle Orthodontist, vol. 68, no. 6, pp. 557-562, 1998.

[27] T. Masumoto, I. Hayashi, A. Kawamura, K. Tanaka, and K. Kasai, "Relationships among facial type, buccolingual molar inclination, and cortical bone thickness of the mandible," European Journal of Orthodontics, vol. 23, no. 1, pp. 15-23, 2001.

[28] M. Motoyoshi, T. Yoshida, A. Ono, and N. Shimizu, "Effect of cortical bone thickness and implant placement torque on stability of orthodontic mini-implants," International Journal of Oral and Maxillofacial Implants, vol. 22, no. 5, pp. 779-784, 2007.

[29] H.-J. Kim, H.-S. Yun, H.-D. Park, D.-H. Kim, and Y.-C. Park, "Soft-tissue and cortical-bone thickness at orthodontic implant sites," The American Journal of Orthodontics and Dentofacial Orthopedics, vol. 130, no. 2, pp. 177-182, 2006.

[30] C. Luzi, C. Verna, and B. Melsen, "Guidelines for success in placement of orthodontic mini-implants," Journal of Clinical Orthodontics, vol. 43, no. 1, pp. 39-44, 2009.

[31] E. J. W. Liou, P.-H. Chen, Y.-C. Wang, and J. C.-Y. Lin, "A computed tomographic image study on the thickness of the infrazygomatic crest of the maxilla and its clinical implications for miniscrew insertion," American Journal of Orthodontics and Dentofacial Orthopedics, vol. 131, no. 3, pp. 352-356, 2007.

[32] S. Baumgaertel, "Cortical bone thickness and bone depth of the posterior palatal alveolar process for mini-implant insertion in adults," American Journal of Orthodontics and Dentofacial Orthopedics, vol. 140, no. 6, pp. 806-811, 2011.

[33] S. Baumgaertel, "Quantitative investigation of palatal bone depth and cortical bone thickness for mini-implant placement in adults," The American Journal of Orthodontics and Dentofacial Orthopedics, vol. 136, no. 1, pp. 104-108, 2009.

[34] G. Lemieux, A. Hart, C. Cheretakis et al., "Computed tomographic characterization of mini-implant placement pattern and maximum anchorage force in human cadavers," The American Journal of Orthodontics and Dentofacial Orthopedics, vol. 140, no. 3, pp. 356-365, 2011.

[35] D.-H. Kim, J.-W. Lee, K.-S. Cha, and D.-H. Chung, "Consideration of maxillary sinus bone thickness when installing miniscrews," Korean Journal of Orthodontics, vol. 39, no. 6, pp. 354-361, 2009. 
[36] L. Ardekian, E. Oved-Peleg, E. E. Mactei, and M. Peled, "The clinical significance of sinus membrane perforation during augmentation of the maxillary sinus," Journal of Oral and Maxillofacial Surgery, vol. 64, no. 2, pp. 277-282, 2006.

[37] P. I. Brånemark, R. Adell, T. Albrektsson, U. Lekholm, J. Lindström, and B. Rockler, "An experimental and clinical study of osseointegrated implants penetrating the nasal cavity and maxillary sinus," Journal of Oral and Maxillofacial Surgery, vol. 42, no. 8, pp. 497-505, 1984.

[38] G. M. Raghoebar, R. H. Batenburg, N. M. Timmenga, A. Vissink, and H. Reintsema, "Morbidity and complications of bone grafting of the floor of the maxillary sinus for the placement of endosseous implants," Mund-, Kiefer- und Gesichtschirurgie, vol. 3, pp. S65-S69, 1999.

[39] B. T. Brettin, N. M. Grosland, F. Qian et al., "Bicortical vs monocortical orthodontic skeletal anchorage," American Journal of Orthodontics and Dentofacial Orthopedics, vol. 134, no. 5, pp. 625-635, 2008.

[40] C. L. Fernandes, "Volumetric analysis of maxillary sinuses of Zulu and European crania by helical, multislice computed tomography," Journal of Laryngology and Otology, vol. 118, no. 11, pp. 877-881, 2004. 


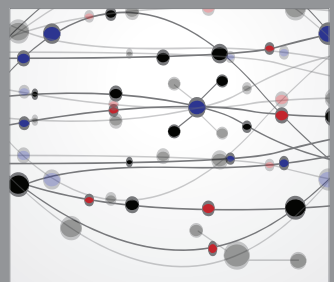

The Scientific World Journal
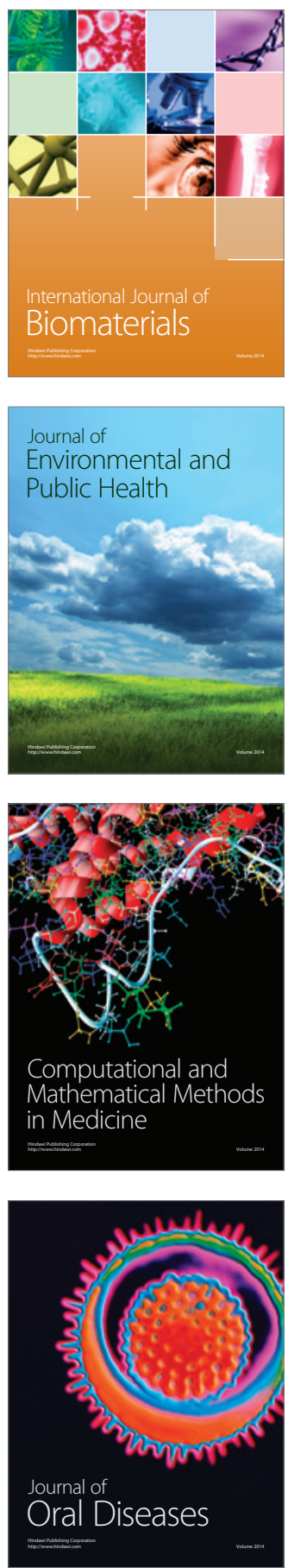
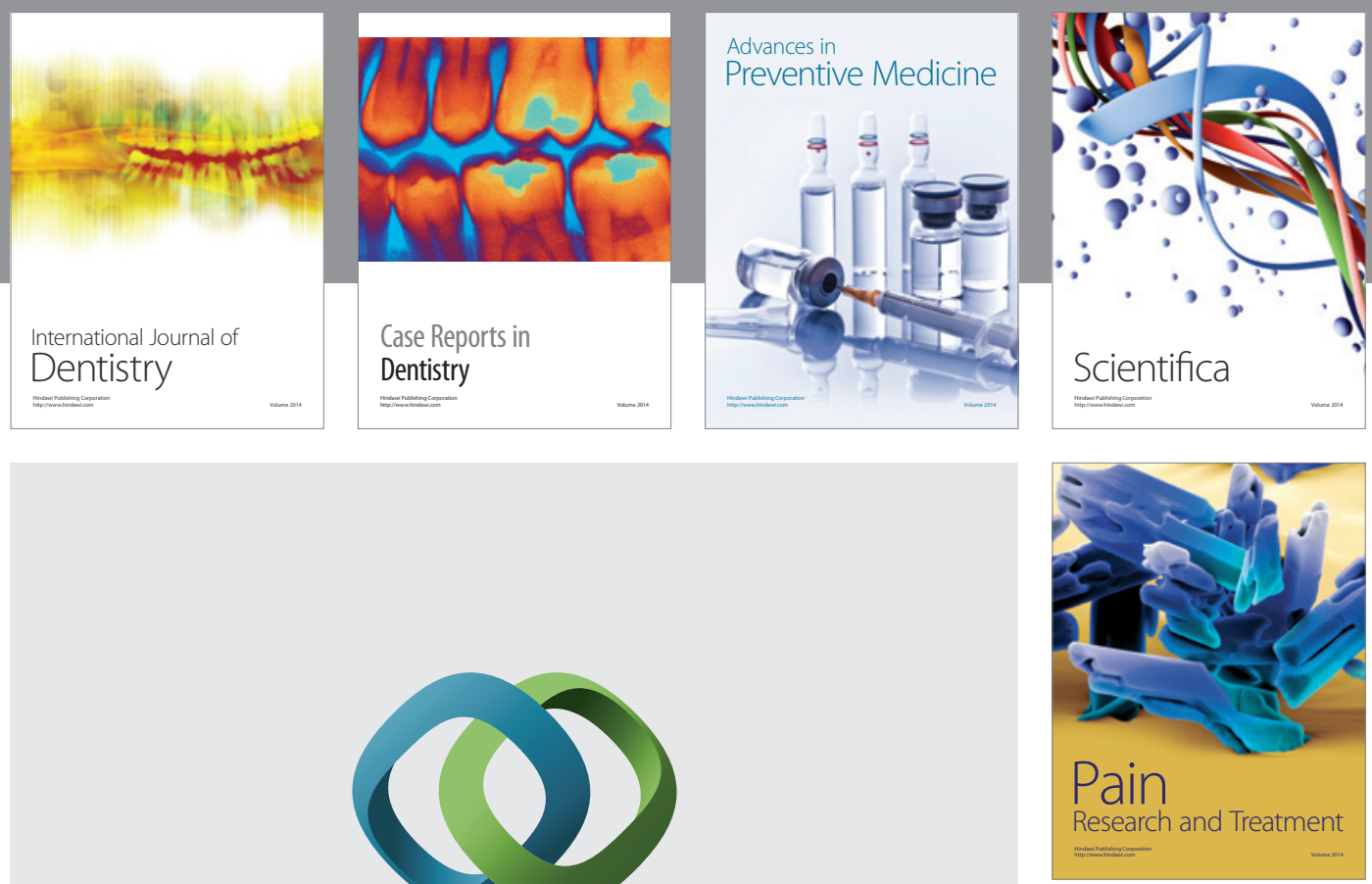

\section{Hindawi}

Submit your manuscripts at

http://www.hindawi.com
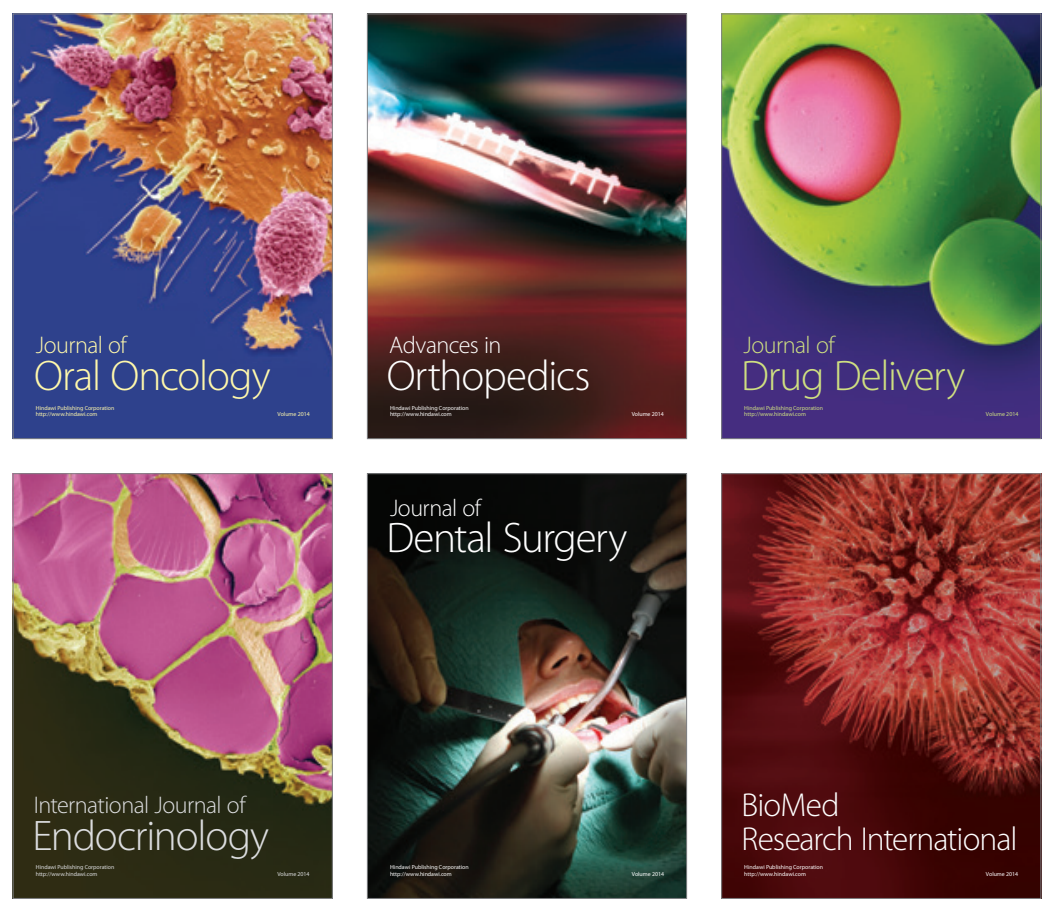

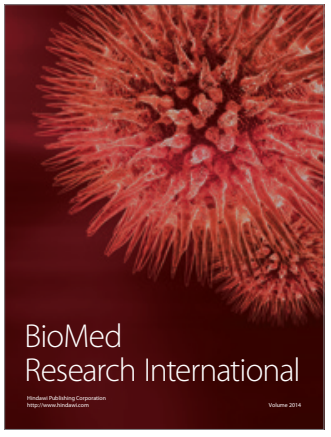

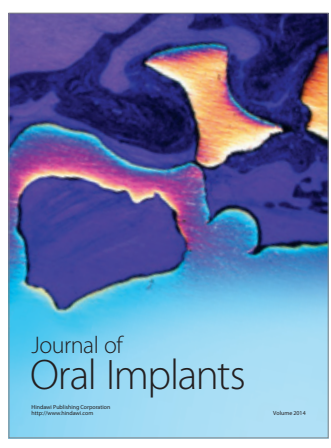
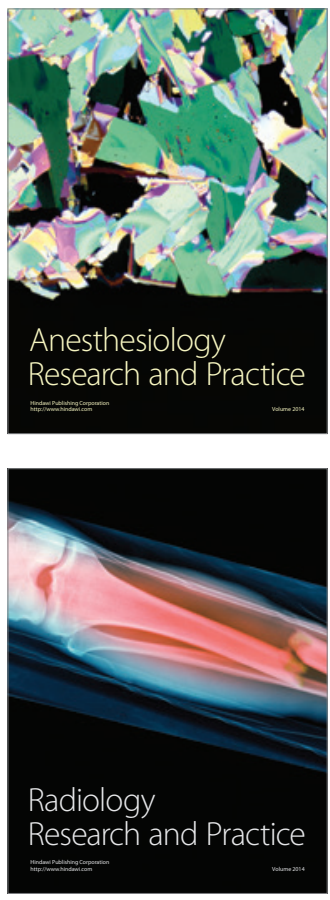